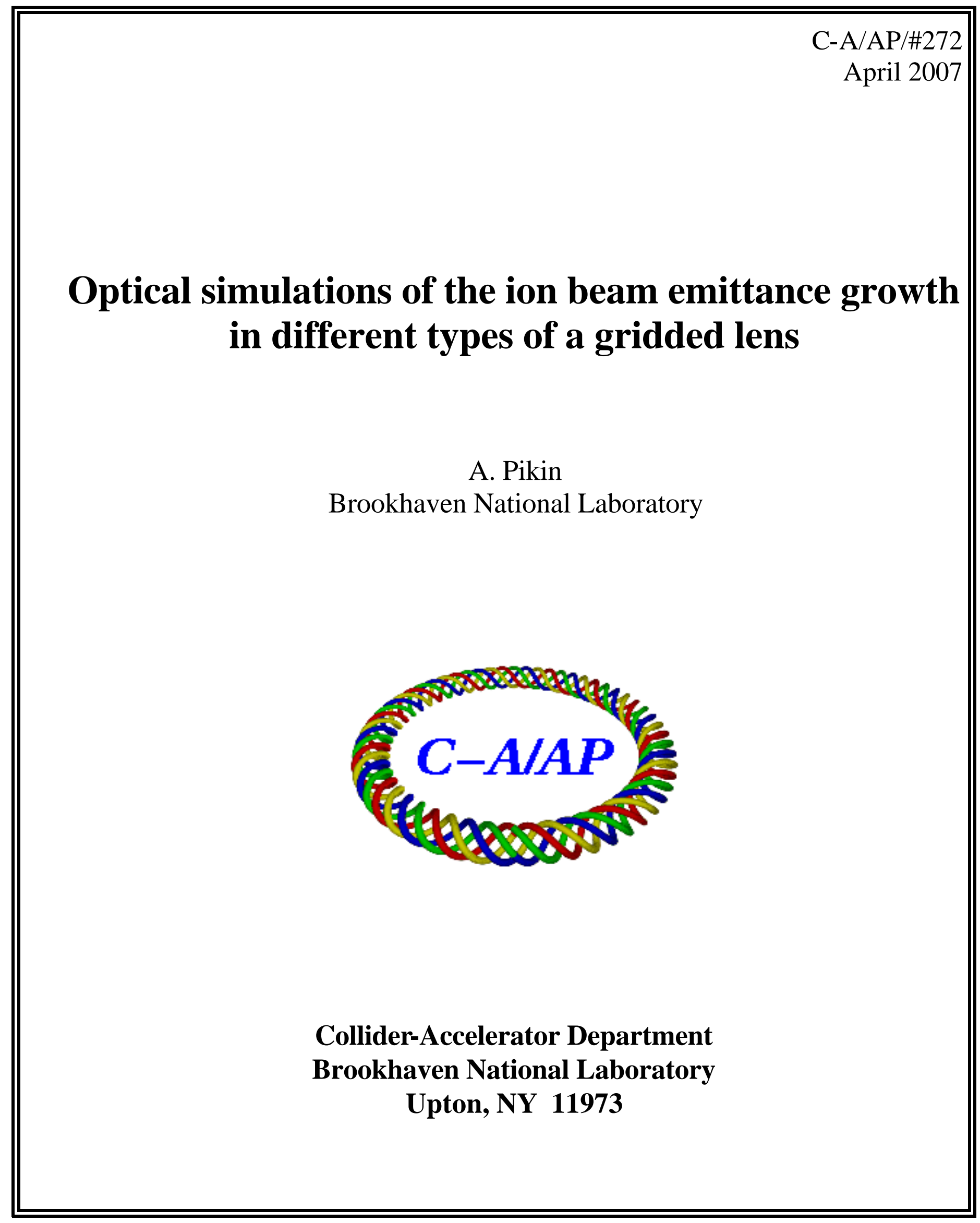




\title{
Optical simulations of the ion beam emittance growth in different types of a gridded lens
}

\author{
A. Pikin
}

A gridded lens has an attractive property of either focusing or defocusing the charged particles beam. And since both sides of such lens have the same direction of radial force it also requires lower voltage for the same focusing strength than an einzel lens. Focusing properties and emittance growth have been studied for different concepts of gridded lens (different combinations of grids and electrodes). Simulations have been done with a parallel ion beam with zero current. The resulting emittance therefore can be interpreted as emittance growth in a lens. These data can be used as a guide for selecting a proper lens for required application and for preliminary assessment of the existing lens. The simulations have been done with 2-dimensional program TRAK [1] using 500 ion trajectories parallel to the z-axis (zero emittance). Ion beam mass is 197, ion charge is +32 , initial ion energy is $17.5 \mathrm{keV}^{*} \mathrm{q}$. The axially symmetric model of the grid itself consists of a set of concentric rings made of dimensionless wires separated radially by 1.0 $\mathrm{mm}$. The different lens geometries are compared for a fixed focal distance $(279.5 \mathrm{~mm})$ from the median plane of the lens. The position of crossover was defined as an average distance from the lens median plane to the crossing of the trajectories with z-axis.

\section{Einzel gridded lens}

This type of lens consists of an isolated cylindrical central electrode and 2 grounded grids on both sides of the electrode. There are several publications on applications of such lens $[2,3]$. The geometry of such lens is presented in Fig. 1.

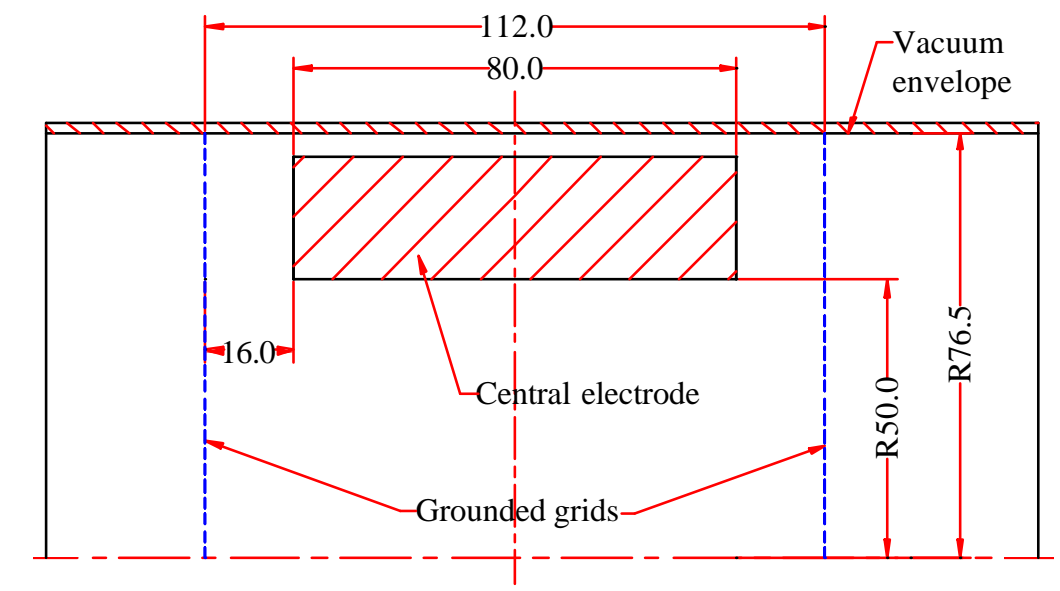

Fig. 1. Geometry of a gridded einzel lens with $\mathrm{ID}=100.0 \mathrm{~mm}$, which can fit inside 6" vacuum pipe.

The electric field distribution for this lens is presented in Fig. 2. 


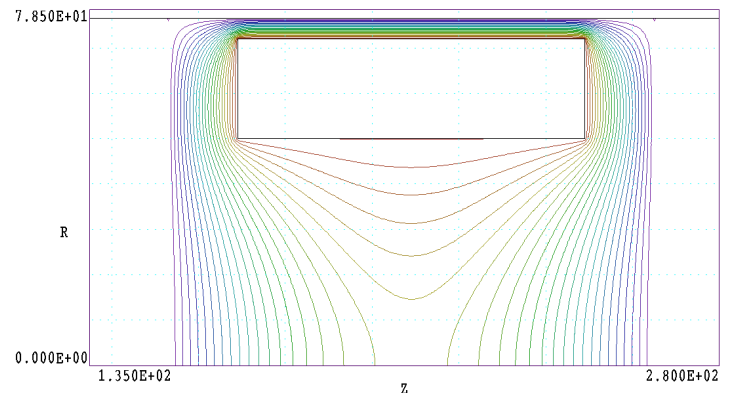

a

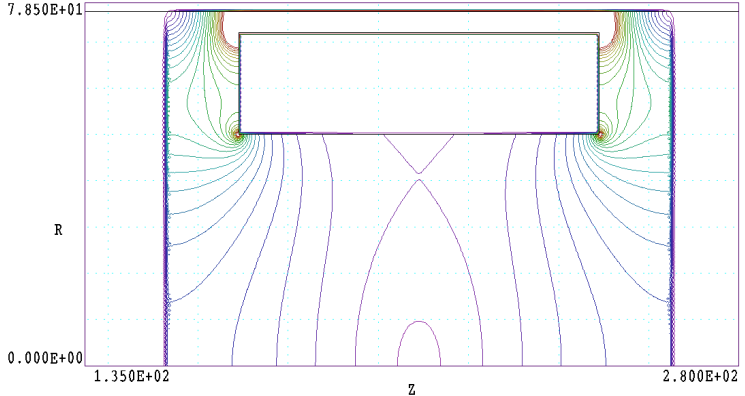

$\mathrm{b}$

Fig. 2. Electrostatic field distribution inside gridded einzel lens: a- equipotential lines, belectric field lines.

The simulated trajectories of an ion beam with diameter $40.0 \mathrm{~mm}$ focused with this lens at a distance of $279.5 \mathrm{~mm}$ from a median plane are presented in Fig. 3. The picture is expanded in vertical direction for clarity.

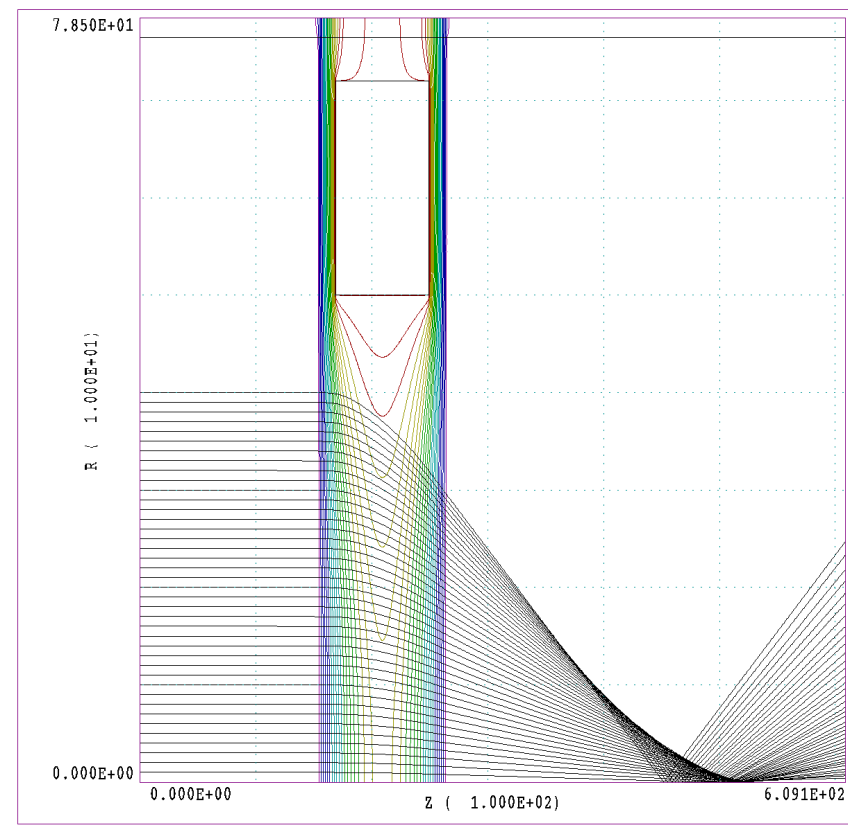

$$
\begin{aligned}
& \text { Total orbits: } 41 \\
& \text { Plotted range } \\
& \begin{array}{lr}
\text { NorbMin: } \quad 1 \\
\text { NorbMax: } \quad 41
\end{array} \\
& \text { Plot mode: ZR } \\
& \text { Magnification: ON } \\
& \text { orbit range } \\
& \text { XMin: } \quad 0.000 \mathrm{E}+00 \\
& \text { XMax: } \quad 0.000 \mathrm{E}+00 \\
& \text { YWax: } \quad 4.000 \mathrm{E}+01 \\
& \text { ZMin: } \quad 0.000 \mathrm{E}+00 \\
& \text { ZMax: } \quad 6.091 \mathrm{E}+02 \\
& \text { RMin: } 0.000 \mathrm{E}+00 \\
& \text { RMax: } \quad 4.000 \mathrm{E}+01 \\
& \text { Plot type: Contour } \\
& \text { Quantity: Phi } \\
& \text { Hinimun value: } \quad 0.000 E+00 \\
& \text { Maximur value: } 4.406 \mathrm{E}+0 \\
& \begin{array}{r}
3.671 \mathrm{E}+02 \\
7.343 \mathrm{E}+02
\end{array} \\
& \begin{array}{l}
1.101 \mathrm{E}+03 \\
1.469 \mathrm{E}+03
\end{array} \\
& 1.836 \mathrm{E}+03 \\
& 2.203 \mathrm{E}+03 \\
& \begin{array}{r}
2.570 \mathrm{E}+03 \\
\square .937 \mathrm{E}+03
\end{array} \\
& \begin{array}{r}
2.9304 \mathrm{~B}+03 \\
\square
\end{array} \\
& \begin{array}{r}
3.671 \mathrm{E}+03 \\
4.0396 \mathrm{0}
\end{array} \\
& \begin{array}{r}
4.039 \mathrm{E}+03 \\
4.406 \mathrm{E}+03
\end{array}
\end{aligned}
$$

Fig. 3. Simulated trajectories of an initial parallel ion beam with zero current passed through the lens with parameters in Fig. 1. The initial energy of ion beam is $17.5 \mathrm{keV}^{*} \mathrm{q}$, ion beam radius is $r_{\text {beam }}=40.0 \mathrm{~mm}$, potential of central electrode $U_{\text {lens }}=4.4 \mathrm{kV}$, distance from the lens median plane to the crossover is $279.5 \mathrm{~mm}$.

The simulated optical properties of this lens are presented in Fig. 4. 


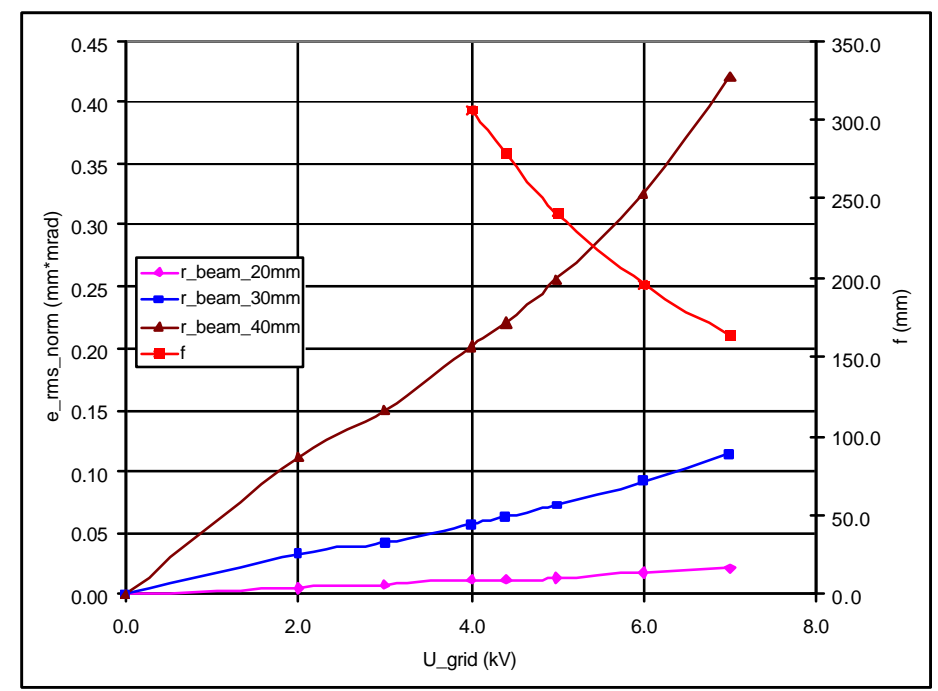

Fig. 4. Dependence of ion beam RMS normalized emittance growth and focal length of gridded einzel lens with parameter Fig. 1 on the potential of central electrode.

A similar lens, which is different from the one in Fig. 1 only by the length of central electrode $(40.0 \mathrm{~mm}$ instead of $80.0 \mathrm{~mm})$, the other parameters are the same (ID of central electrode, distances from the electrode to grids, ID of vacuum pipe) was also simulated and the cumulative graph for both lenses focusing the ion beam at the same distance is presented in Fig. 5.

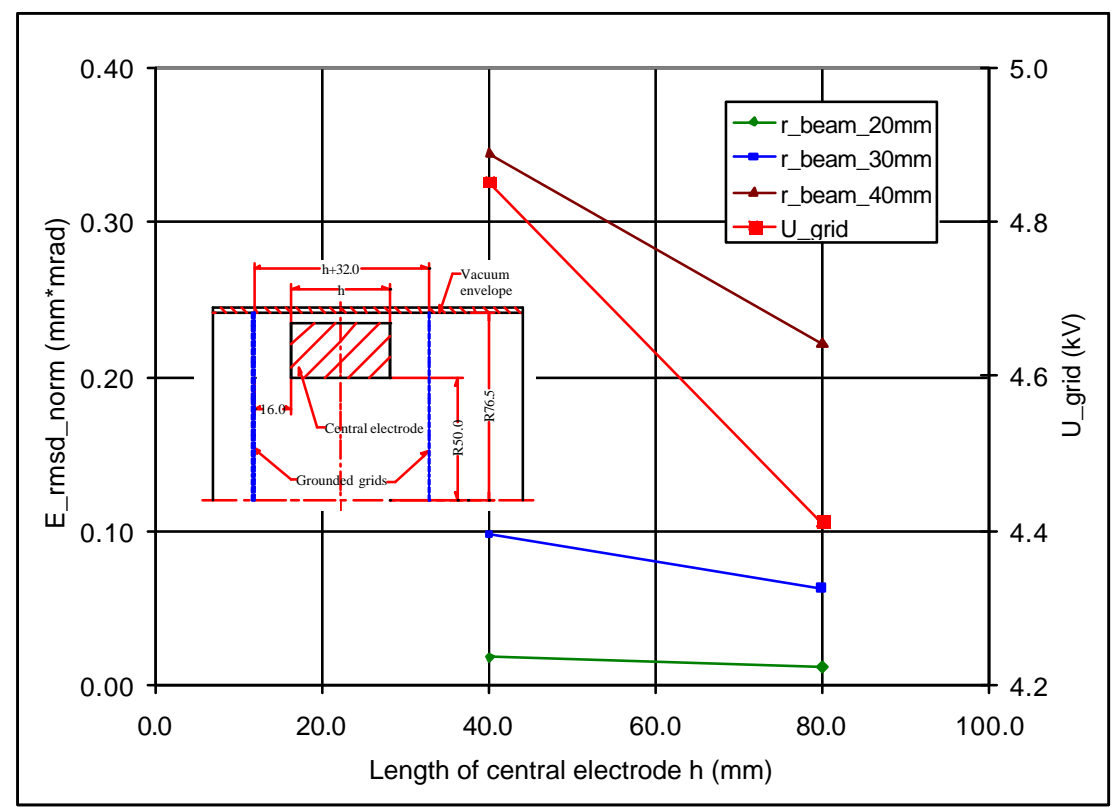

Fig. 5. Dependence of the rms normalized emittance growth and voltage required to focus a parallel ion beam with zero current and energy $\mathrm{E}_{\mathrm{ion}}=17.5 \mathrm{keV}^{*} \mathrm{q}$ at a distance of $279.5 \mathrm{~mm}$ from the lens median plane on the length of central electrode " $h$ " with other parameters in Fig. 1. 
One can see that both the ion beam emittance growth and the voltage required to focus the ion beam on the same distance decrease with increased length of the central electrode. The possible reason of the emittance increase with decreasing the gap between grids is "flattening" the equipotential surfaces in a near-axis region as fields of both grids push against each other and making these surfaces steeper in a region with larger radius. To reduce the spherical aberration of this type of lens the grids should be sufficiently separated from each other so the interaction of their fields is negligible.

\section{Gridded lens with grounded cylindrical electrodes on both sides of a single grid.}

This type of lens consists of a single isolated grid with two field-shaping grounded cylindrical electrodes on both sides. Schematic of this concept is presented in Fig. 6.

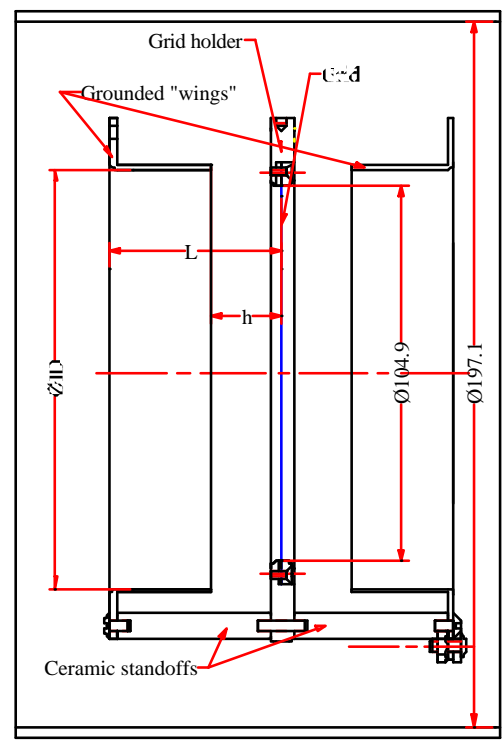

Fig. 6. Geometry of gridded lens with grounded cylindrical electrodes.

2.1 Dependence of the ion beam emittance growth on the gap (h) between grid and closest edge of the grounded cylindrical electrode.

Other parameters of this lens:

- Inner diameter of the cylindrical electrode ID=117.4 mm

- Opened diameter of the grid $\mathrm{D}_{\text {grid }}=104.9 \mathrm{~mm}$

- Distance from grid to the outer side of the cylindrical electrode $\mathrm{L}=48.0 \mathrm{~mm}$ Ion beam energy $\mathrm{E}_{\text {ion }}=17.5 \mathrm{keV} * \mathrm{q}$ ( $\mathrm{q}$ - ion charge state, we used ions $\mathrm{Au}_{197}{ }^{+32}$ ). Two beam radii have been simulated: $30.0 \mathrm{~mm}$ and $40.0 \mathrm{~mm}$.

The results of this set of simulations are presented in Fig. 7. 


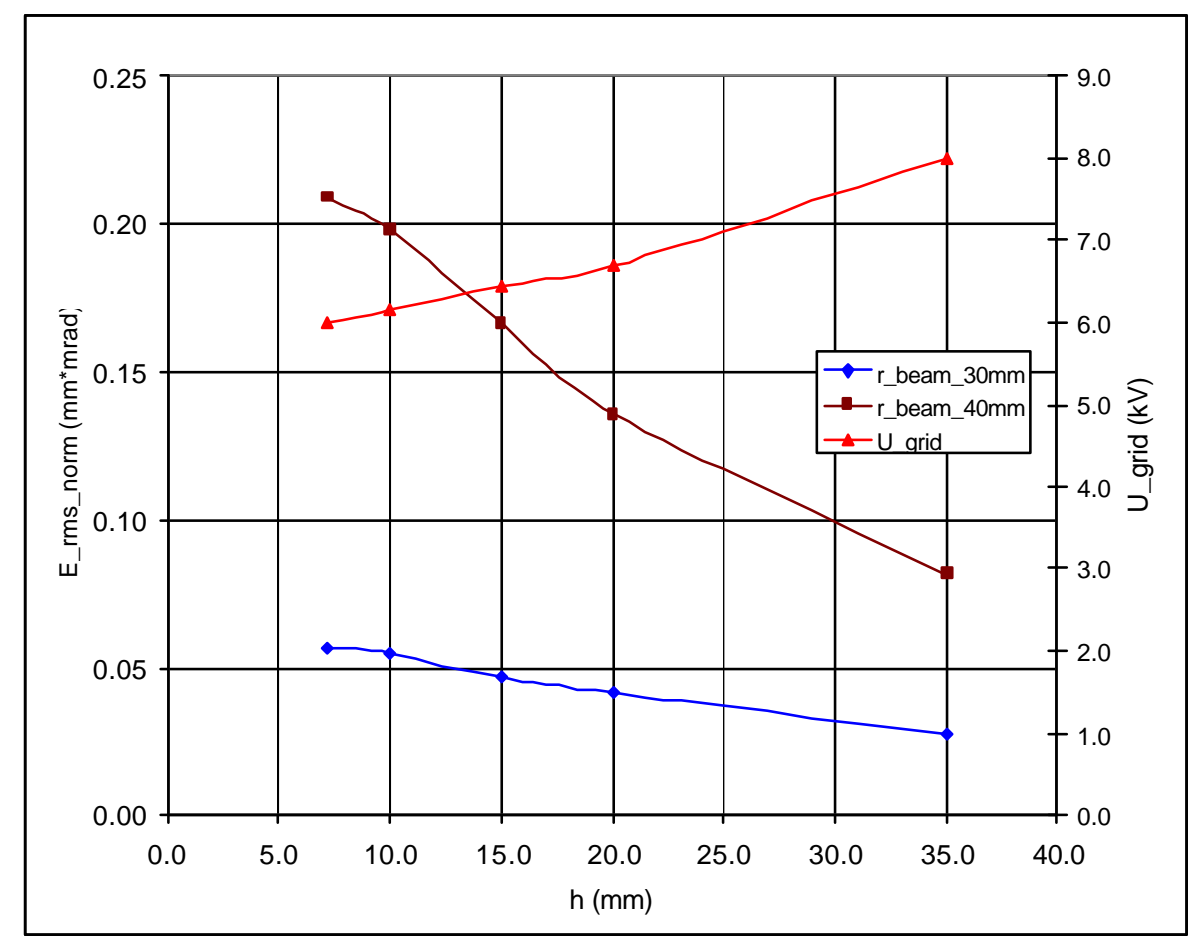

Fig. 7. Dependence of the ion beam emittance growth and voltage required to focus the ion beam on a distance of $279.5 \mathrm{~mm}$ from the grid on the distance " $h$ " from the grid to the closest edge of the grounded electrodes.

These results demonstrate that with increased distance " $h$ " the emittance of the ion beam decreases and this decrease is more pronounced for larger beam radii. The factor of required grid voltage increase to maintain the same focusing strength of the lens is lower than the factor of reducing the emittance for the same increase of distance " $h$ ".

2.2 Dependence of the ion beam emittance growth on the inner diameter ID of the grounded cylindrical electrode.

Parameters of the lens:

- Distance from grid to closest edge of cylindrical electrode: $\mathrm{h}=10.0 \mathrm{~mm}$

- Distance from grid to outer edge of cylindrical electrode: $\mathrm{L}=48.0 \mathrm{~mm}$

Beam parameters are the same is in a previous case.

The results are presented in Fig. 8. 


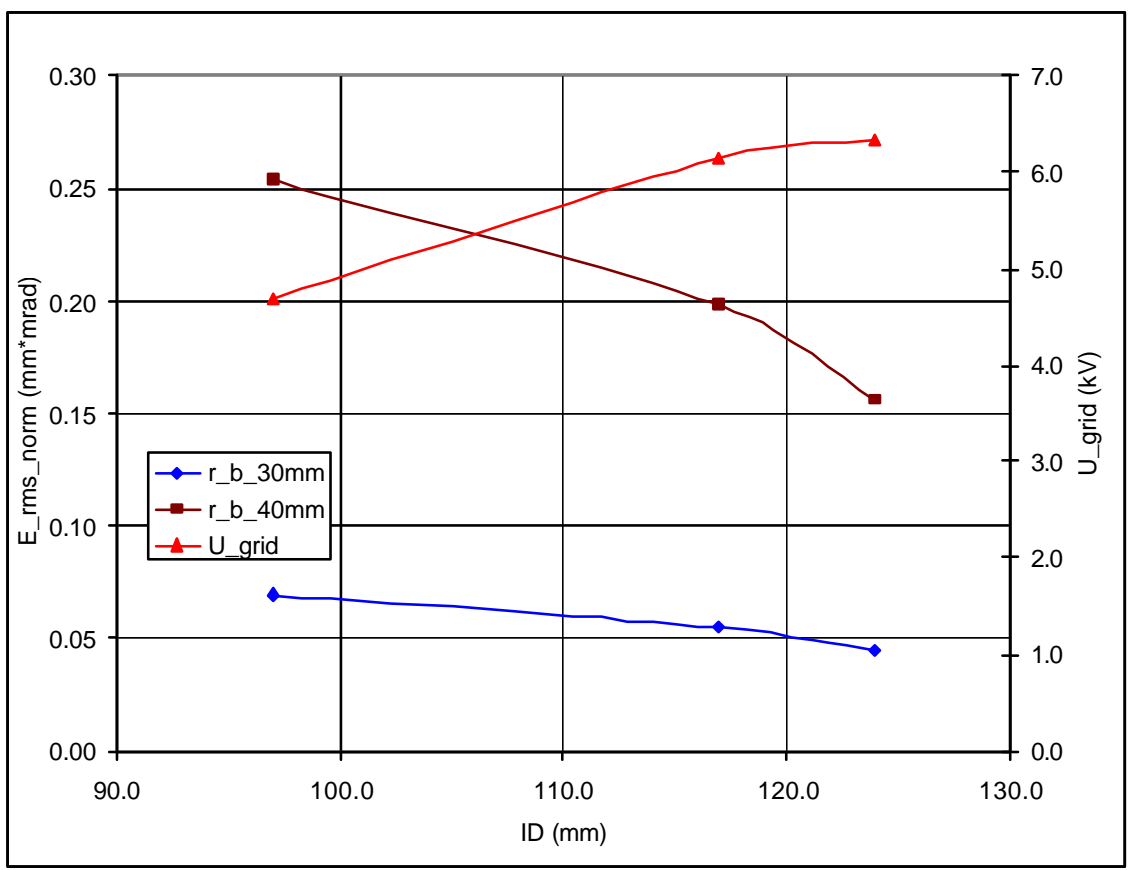

Fig. 8. Dependence of the ion beam emittance growth and voltage required to focus the ion beam on a distance of $279.5 \mathrm{~mm}$ from the grid on inner diameter of grounded cylindrical electrode.

As expected with larger ID of cylindrical electrode the emittance growth is lower although this dependence is not as strong as h-dependence.

1.3 Dependence of emittance growth on a distance from grid to the outer edge of the grounded cylindrical electrode "L".

For 2 values of L: $28 \mathrm{~mm}$ and $48 \mathrm{~mm}$ (ID=117.4 mm, h=10.0 mm) no visible difference in emittance growth has been noticed.

\section{Gridded lens with no special grounded electrodes, vacuum wall serves as a field terminator.}

\subsection{Rounded grid holder.}

The geometry of this concept is presented in Fig. 9. The edges of the grid holder are rounded off with radius $\mathrm{R}$ and its internal surface has the shape of toroid with small radius $R$ and the width of the holder is $h=2 R$.

Lens parameters:

- ID of the grid (opened diameter): $\mathrm{ID}_{\text {grid }}=104.0 \mathrm{~mm}$

- ID of the grounded vacuum wall: $\mathrm{ID}_{\text {wall }}=153.0 \mathrm{~mm}$

These dimensions are picked for the lens, which can fit inside a 6" vacuum pipe with $1 / 2$ " standoff insulators. 


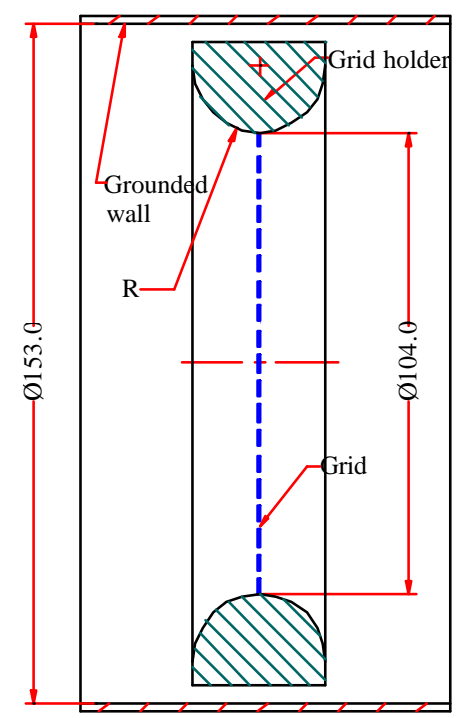

Fig. 9. Geometry of the gridded lens without dedicated grounded terminators.

The simulated ion trajectories for $\mathrm{R}=10.0 \mathrm{~mm}$ are presented in Fig. 10 (the original picture is expanded vertically for more clarity).

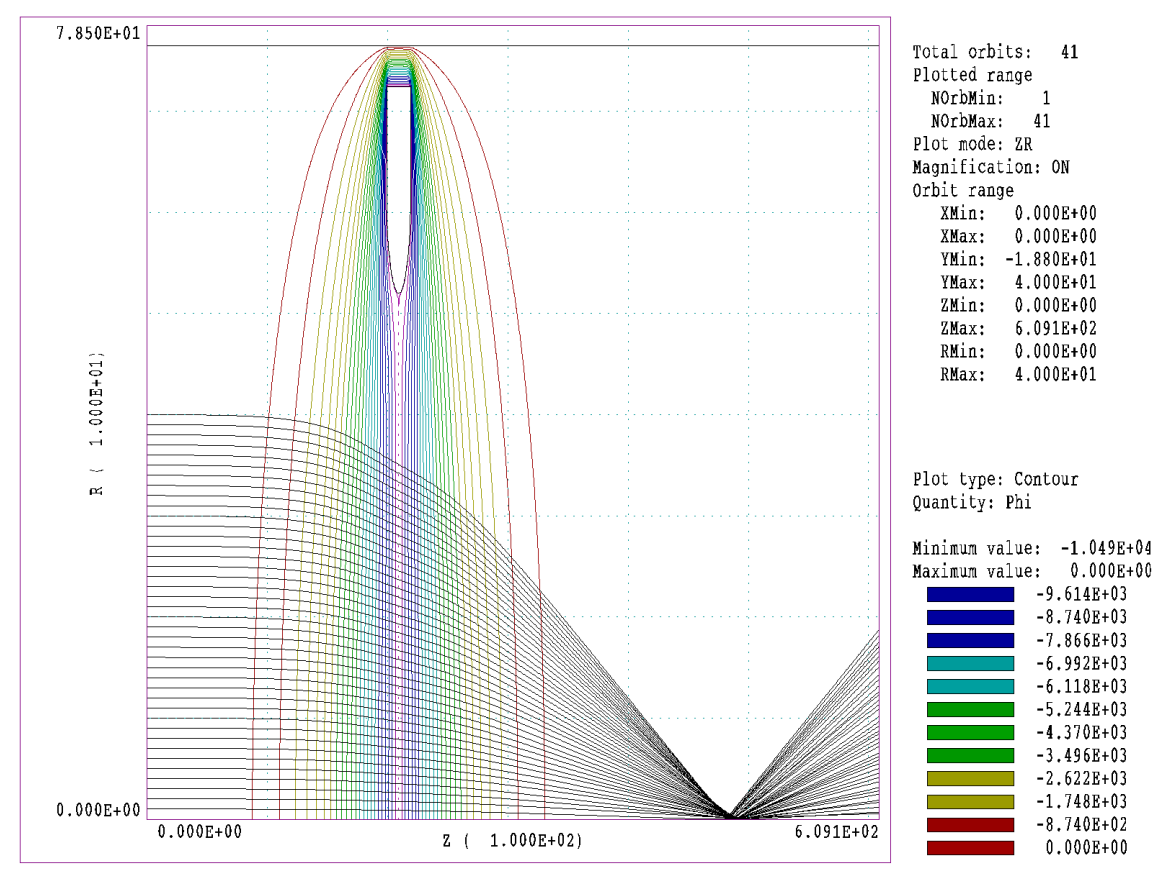

Fig. 10. Simulated trajectories for gridded lens presented on Fig.4 $(R=10)$. Radius of ion beam $\mathrm{R}_{\text {beam }}=40.0 \mathrm{~mm}, \mathrm{E}_{\text {ion }}=17.5 \mathrm{keV}^{*} \mathrm{q}, \mathrm{U}_{\text {grid }}=-10.5 \mathrm{kV}$, focal length $\mathrm{f}=279.5 \mathrm{~mm}$.

The results of simulations of this gridded lens concept are presented in Fig. 6. 


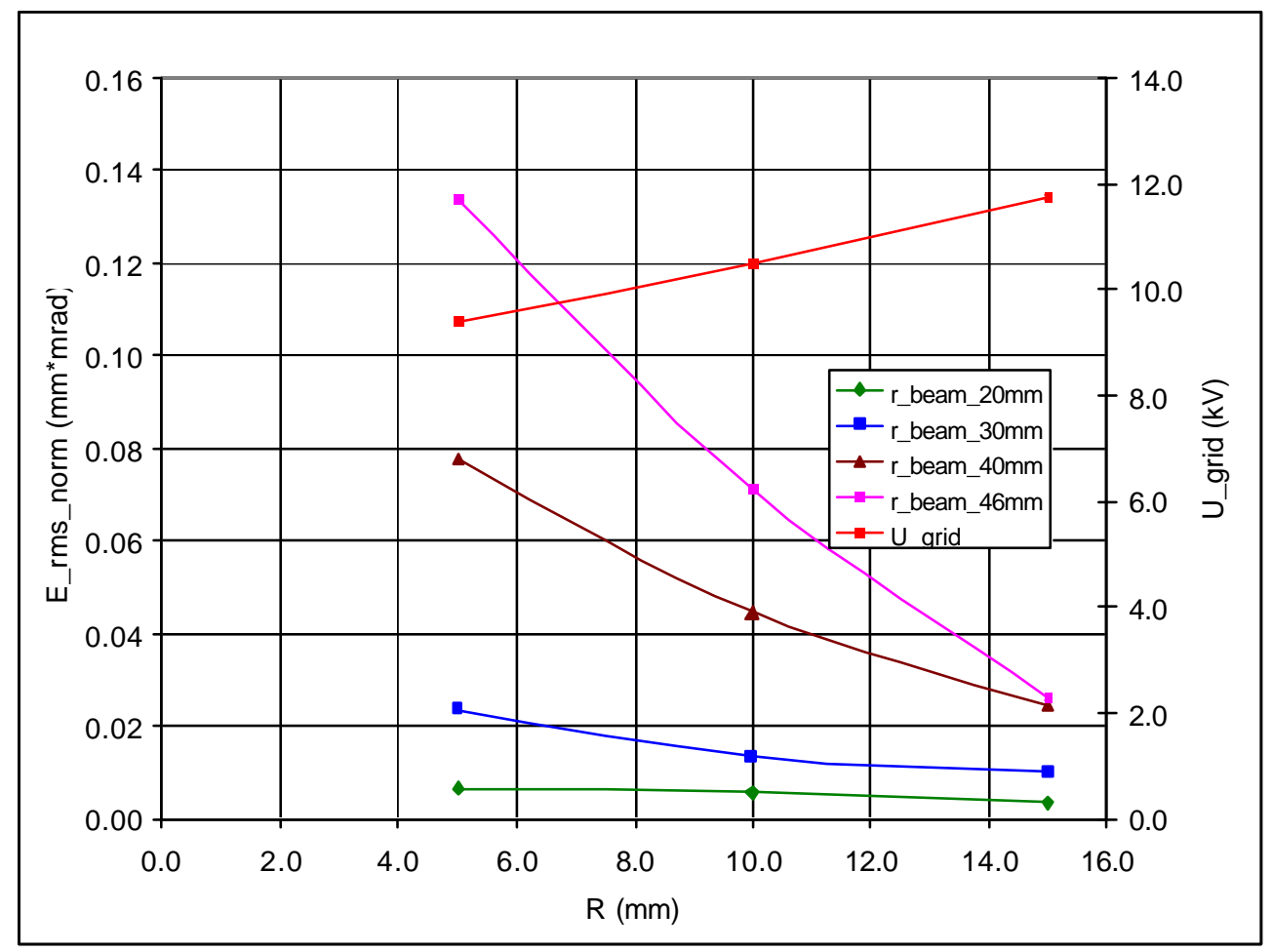

Fig. 11. Dependence of the ion beam emittance growth and voltage required to focus the ion beam on a distance of $279.5 \mathrm{~mm}$ from the grid on a half width of the grid holder $(\mathrm{R})$.

2.2 Rectangular grid holder

The geometry of this lens is very similar to the previous case, except the grid holder is not rounded, it is rectangular. The geometry of this lens is presented in Fig. 12.

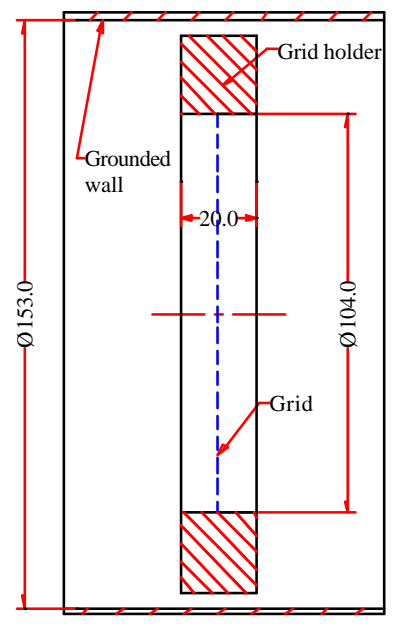

Fig. 12. Gridded lens with rectangular grid holder. 
The result of this lens analysis is presented in Fig. 13.

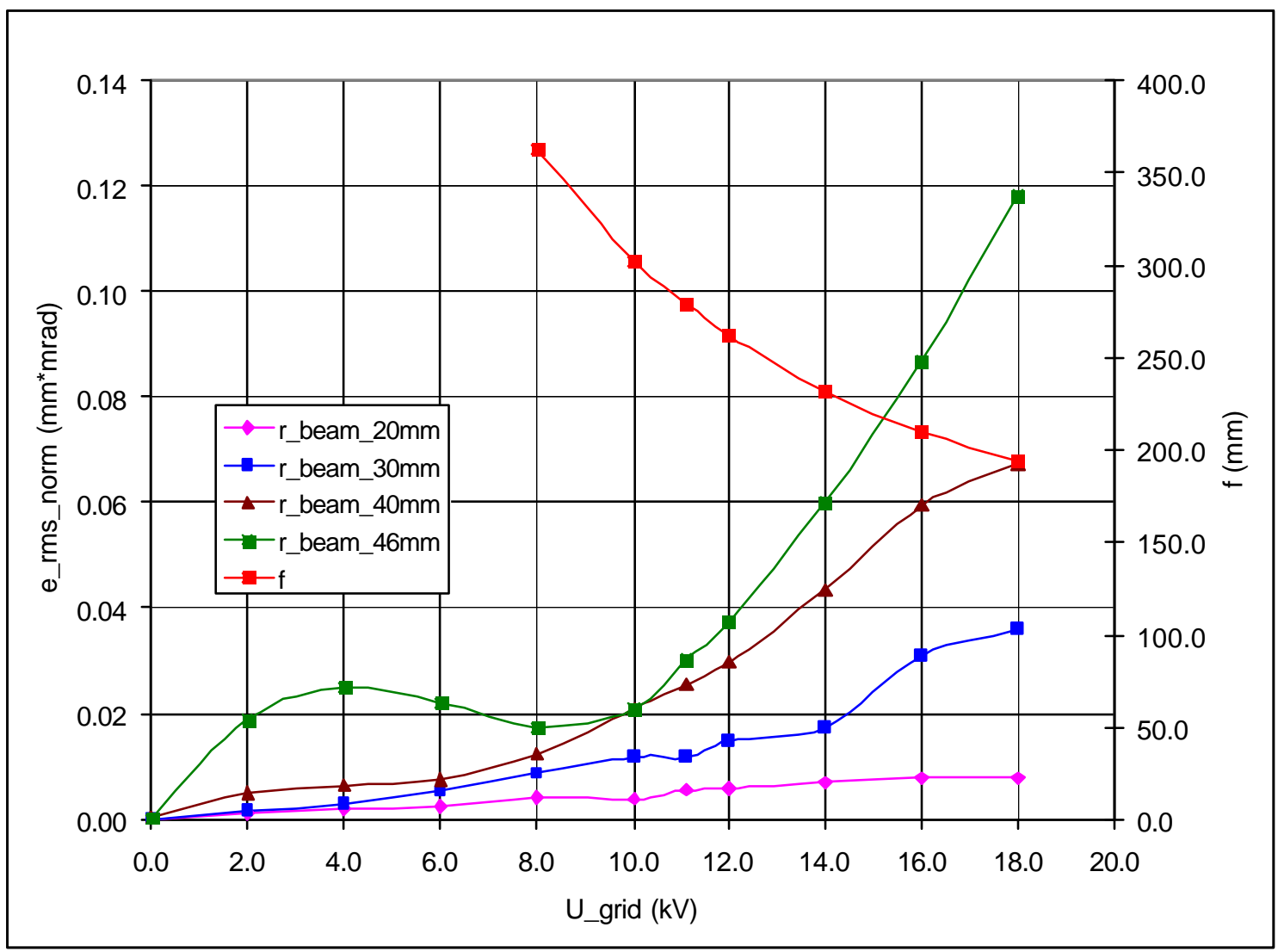

Fig. 13. Dependence of rms normalized emittance growth and focal distance of the lens with rectangular grid holder and parameters presented in Fig. 12.

Both focusing properties and aberrations of this lens are very similar to the gridded lens with rounded grid holder. The rectangular shape mostly affects the very peripheral trajectories by making the energy dependence of emittance non-linear for beam diameter close to the aperture ID (but not larger than for rounded gridded holder in a studied voltage range).

\section{Resume}

1. The simulations of different types of gridded lens with idealized parallel beam with zero current demonstrate that a gridded lens with no dedicated grounded electrodes produces much lower emittance growth of the ion beam than a gridded einzel lens with similar ID or a gridded lens with single grid and grounded cylindrical electrodes.

2. The emittance growth produced by such lens decreases with increased width of the grid holder within simulated range of this width. A further optimization of the shape of the grid holder might reduce the spherical aberrations even more. 
3. Even that the design of the gridded lens does not have dedicated grounded electrodes, the application of this lens presumes that the lens is mounted on the axis of the grounded vacuum pipe and that the close vicinity of this lens has axial symmetry.

A possible design of the modular gridded lens mounted on a standard 2-sided 8" CF flange is presented in Fig. 14. Such lens can be inserted in any position of the beam line between existing flanges with minimal axial space requirement.

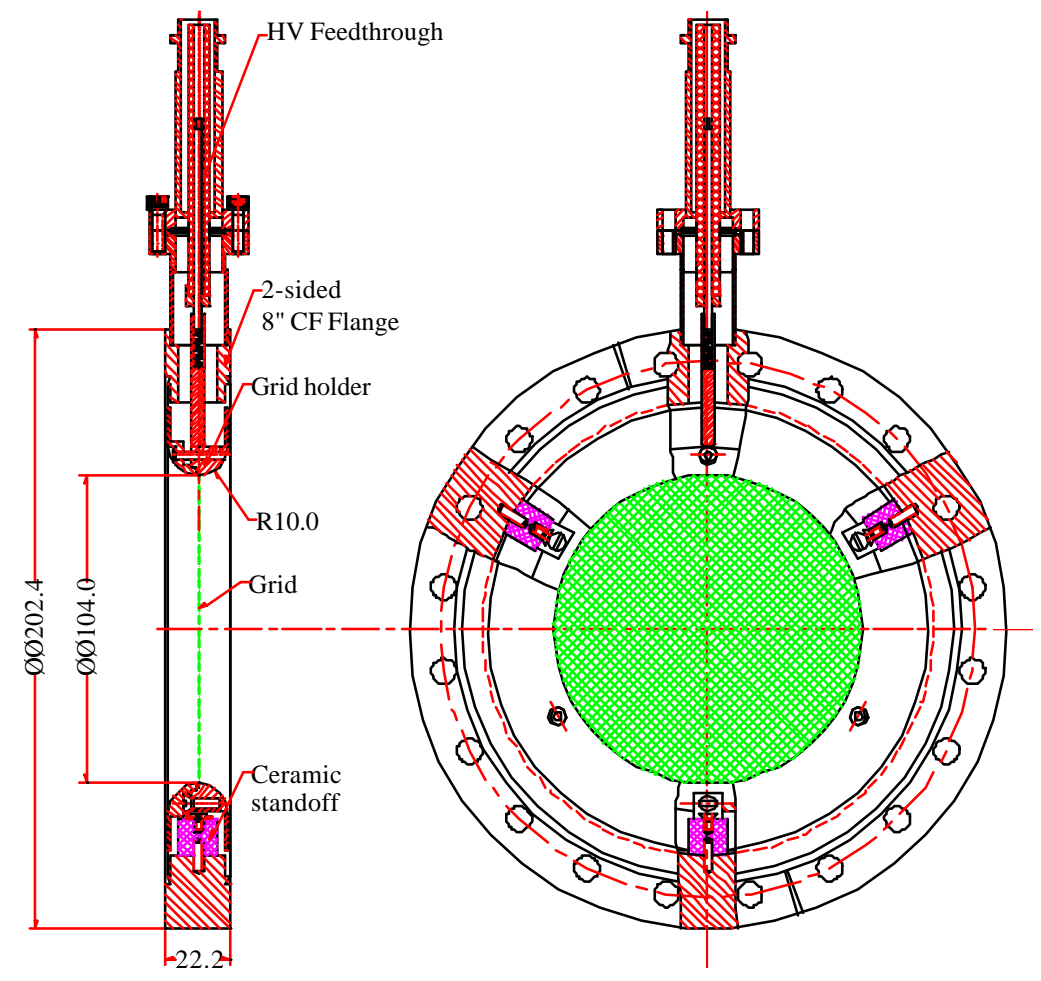

Fig. 14. A possible design of the modular gridded lens.

\section{References:}

1. http://www. fieldp.com/

2. Developments of gridded ion lenses, J. W. Johnson, PAC1965 p.765 -768. http://epaper.kek.jp/p65/PDF/PAC1965_0765.PDF

3. Use of electrostatic gridded lenses in the laser ion source at CERN. V. Derbilov, P. Ostroumov, etc. Proceedings of 26 International Workshop on Charged Particles Linear Accelerators, 1999, p. 313, http://www.kipt.kharkov.ua/conferences/ihepnp/16workshop/PDF/al9_313.pdf 